\title{
Adiciones a la fauna de bivalvos del mar peruano
}

\author{
Additions to the bivalve fauna of the Peruvian sea
}

\section{Carlos Paredes ${ }^{1,2}$, Franz Cardoso ${ }^{1}$, Leonardo Romero ${ }^{1}$ y Remy Canales ${ }^{1}$}

1 Laboratorio de Biología y Sistemática de Invertebrados Marinos, Tacultad dr Fnivertad de Ciencias Biológicas, Universidad Nacional Mayor de San Marcos, Apdo. 11-0058, Lima, 11, Perú. Email Carlos Paredes: cparedesq@unmsm.edu.pe

2 Departamento de Malacología, Museo de Historia Natural, Universidad Nacional Mayor de San Marcos, Apdo. 14-0434, Lima 14, Perú.

Presentado: 01/11/2011 Aceptado: $\quad 07 / 02 / 2012$ Publicado online: 01/10/2012

\section{Resumen}

Se registran por primera vez para el mar peruano 10 especies de bivalvos: Ennucula panamina (Dall, 1908), Nuculana (Nuculana) loshka, (Dall, 1908), Saccella laeviradius (Pilsbry \& Lowe, 1932), Liralucina approximata (Dall, 1901), Carditamera radiata (G. B. Sowerby I, 1833), Carditella (Carditella) naviformis (Reeve, 1843); Macoma (Psammacoma) elytrum Keen, 1958, Psammotreta (Psammotreta) pura (Gould, 1853), Sphenia fragilis (H. Adams \& A. Adams, 1854) y Caryocorbula amethystina Olsson, 1961. Se informa sobre la distribución, el hábitat y comentarios relevantes sobre cada una de las especies.

Palabras claves: Mollusca, Bivalvia, Protobranchia, Heterodonta, nuevos registros, Perú.

\section{Abstract}

The following species of Bivalvia are recorded for the first time for Peruvian sea: Ennucula panamina (Dall, 1908), Nuculana (Nuculana) loshka, (Dall,1908), Saccella laeviradius (Pilsbry \& Lowe, 1932), Liralucina approximata (Dall, 1901), Carditamera radiata (G. B. Sowerby I, 1833), Carditella (Carditella) naviformis (Reeve, 1843); Macoma (Psammacoma) elytrum Keen, 1958, Psammotreta (Psammotreta) pura (Gould, 1853), Sphenia fragilis (H. Adams \& A. Adams, 1854) y Caryocorbula amethystina Olsson, 1961. Se informa sobre la distribución, el hábitat y comentarios relevantes sobre cada una de las especies.

Keywords: Mollusks, Bivalvia,Protobranchia, Heterodonta, nuevos registros, Perú.

\section{Introducción}

El conocimiento de la diversidad de moluscos marinos del Perú es incompleto, presentándose varios vacios de información enumerados desde la década pasada (Ramírez et al. 2003), de los cuales podemos señalar a la falta de exploración sistemática tanto de ambientes como de grupos taxonómicos o morfológicos (e.g. micromoluscos) como vacios persistentes.

Nosotros desde hace varios años venimos trabajando en el inventario y la determinación taxonómica actualizada de los moluscos marinos (e.g. Paredes et al. 1998, 1999, 2005, 2010; Paredes y Cardoso, 1998, 2001a, 2001b, 2003, 2007, 2008, 2009). El presente trabajo actualiza la lista de los moluscos marinos del Perú, como parte de la elaboración del Catálogo Ilustrado de Moluscos Bivalvos del Mar Peruano. En esta oportunidad se da a conocer el hallazgo de 3 especies de Bivalvos de la subclase Protobranchia, y 7 especies de la Subclase Heterodonta, que son registradas por primera vez para el mar peruano.

\section{Material y métodos}

La mayor parte del material revisado procede de colectas realizadas en aguas tropicales del mar peruano, frente a los Departamentos de Tumbes y Piura. Solamente Carditella (Carditella) naviformis (Reeve, 1843) fue colectada en aguas de la Provincia Peruana.

El material biológico fue fijado en el campo o en el laboratorio, utilizando formol al 7\% neutralizado con bórax.

Para su estudio, se lavaron las muestras en agua corriente y luego se utilizó alcohol al 70\% para su conservación. El material se encuentra depositado en el Laboratorio de Biología y Sistemática de Invertebrados Marinos, Facultad de Ciencias Biológicas, Universidad Nacional Mayor de San Marcos (LaBSIM). El ordenamiento supraespecífico está basado en Skoglund (2001) y para la determinación taxonómica se utilizó la bibliografía especializada. También se realizaron consultas a especialistas del Museo de Santa Bárbara, California .

\section{Taxonomía}

Subclase PRotobranchia

Orden NuCuloida Dall, 1889

Superfamilia Nuculoidea Gray, 1824

FAMILIA NuCULIDAE GRAY, 1824

Genero Ennucula Iredale, 1931

Ennucula panamina (Dall, 1908)

Figura 1

Nucula panamina Dall, 1908: 219, 368, pl. 6, fig. 11; Hertlein \& Strong, 1940: 386; Keen, 1971: 26, fig. 13.

Nucula (Leionucula) panamina, Bernard, 1983: 10.

Ennucula panamina, Skoglund, 2001: 5.

Ennucula cardara, Paredes \& Cardoso, 2001a: 7, fig. 2.

Concha sólida, subtriangular, umbo cerca del extremo posterior con pico opistogirado, borde ánterodorsal suavemente arqueado con extremo redondeado, extremo posterior anguloso y borde ventral arqueado; charnela taxodonta, con 20 dientes anteriores y 10 posteriores, el condróforo es oblicuo y profundo; color externo blanquecino con periostraco pardo oliváceo, brillante y muy adherente, el cual deja ver finas estrías concéntricas en la conchilla; interior blanco nacarado y bordes de las valvas lisos. Longitud, 22,0 $\mathrm{mm}$; altura $16,0 \mathrm{~mm}$.

Distribución: $6^{\circ}$ a $31^{\circ} \mathrm{N}$ (Skoglund, 2001).

Localidades: Piura (Frente a Punta Máncora).

Habitat: Fondo arenoso, $551 \mathrm{~m}$.

Material examinado: 1 lote, 2 especímenes (LaBSIM). 
Observaciones: Esta especie fue determinada por P. V. Scott. Nuevo registro para el mar peruano.

Superfamilia Nuculanoidea H. Adams \& A. Adams, 1858

Familia Nuculanidae H. Adams \& A. Adams, 1858

Subfamilia Nuculaninae H. Adams \& A. Adams, 1858 Genero NuCulana LinK, 1807

SUBgenERo NUCULANA, S.S.

\section{Nuculana (Nuculana) loshka (Dall, 1908)}

\author{
Figura 2
}

Leda loshka Dall 1908: 219,376, pl. 17, fig. 2.

Nuculana loshka, Hertlein \& Strong, 1940: 404-405; Keen, 1971: 31. Nuculana (Thestyleda) loshka, Bernard, 1983: 13.

Nuculana (Nuculana) loshka, Skoglund, 2001: 5, como sinónimo. de Nuculana (N.)extenuata (Dall, 1897).

Nuculana (N.)extenuata, Paredes y Cardoso, 2001a, 7, fig. 3.

Concha pequeña, delgada, inflada, con borde anterior redondeado y el lado posterior prolongado para formar un largo rostrum cuyo borde dorsal es ligeramente cóncavo y bicarinado en cada valva, el espacio entre las carinas presenta, cuerdas concéntricas; el área umbonal lleva varias cuerdas concéntricas notorias y en el resto del disco hay finas estrías concéntricas; charnela taxodonta con 18 dientes anteriores y 30 posteriores; el ligamento se inserta oblicuamente en suaves depresiones del condróforo, el cual es una lámina situada bajo los umbos. El periostraco oliváceo cubre la conchilla blanquecina; interior blanco nacarado algo translúcido y los bordes de las valvas lisos. Longitud $17,0 \mathrm{~mm}$; altura $7,0 \mathrm{~mm}$.

Distribución: Golfo de Panamá (Hertlein \& Strong, 1940).

Localidades: Piura (Frente a Isla Foca).

Habitat: Fondo areno fangoso, $864 \mathrm{~m}$.

Material examinado: 1 lote, 1 especimen (LaBSIM).

Observaciones: Esta especie fue determinada por P. V. Scott. Nuevo registro para el mar peruano.

$$
\text { Genero Saccella Woodring, } 1925
$$

Saccella laeviradius (Pilsbry \& Lowe, 1932)

\section{Figura 3}

Leda laeviradius Pilsbry \& Lowe, 1932: 106, pl. 17, fig. 7;Olsson, 1961: 60-61, como sinónimo de Nuculana (Saccella) acrita; Keen, 1971: 29, como sinónimo de Nuculana (Saccella) acrita; Abbott, 1974: 416, como sinónimo de Nuculana (Saccella) acrita; Bernard, 1983: 12, como sinónimo de Nuculana (Saccella) acrita; Skoglund, 2001: 6, como sinónimo de Nuculana (Saccella) acrita.

Nuculana (Saccella) laeviradius, Hertlein \& Strong, 1940: 396-397.

Concha pequeña, translúcida casi equilateral, lado anterior inflado y posterior prolongado en un estrecho rostrum que termina redondeado, el área rostral deprimida y con estrías longitudinales está limitada a cada lado por un lomo redondeado; escultura consiste en nítidas ondas concéntricas que se debilitan al alcanzar el lomo posterior, pero no se aprecia claramente el rayo liso descrito para esta especie, que se extiende desde el pico hasta el hasta el borde inferior; un rayo suave deprimido se extiende desde el pico hasta el borde ventral anterior, y en el límite anterior de este rayo las ondas se levantan formando una línea carinada; la charnela taxodonta presenta 16 dientes anteriores y 15 posteriores, el pico es opistogirado y el resilio pequeño; el periostraco es pardo claro; exterior e interior de la concha blanquecino traslúcido y bordes de las valvas lisos. Longitud $6,2 \mathrm{~mm}$; altura $3,3 \mathrm{~mm}$.

Distribución: Punta Peñasco, Sonora, Mexico, hasta Port Parker, Costa Rica (Hertlein \& Strong, 1940).

Localidades: Piura (Bahía de Paita).

Habitat: Sublitoral, fondo fango arenoso con restos de conchillas, 12-15 brazas (Hertlein \& Strong, 1940).

Material examinado: 1 lote, 1 especimen en fondo arenoso a $35 \mathrm{~m}$ de profundidad (LaBSIM).

Observaciones: Esta especie fue determinada por E. V. Coan. Nuevo registro para el mar peruano.

\author{
Subclase Heterodonta \\ ORDEN VENEROIDA \\ Genero Parvilucina Dall, 1901
}

\section{Liralucina approximata (Dall, 1901)}

\section{Figura 4}

Phacoides (Parvilucina) approximatus Dall, 1901: 813, 828, pl. 39, fig. 4.

Lucina (Parvilucina) approximata, Hertlein \& Strong, 1946: 115116; Olsson, 1961: 214, pl. 31, fig. 7; Keen, 1971: 121, fig. 274; Bernard, 1983: 29.

Parvilucina (Parvilucina) approximata, Abbott, 1974: 459, fig. 5292; Skoglund, 2001: 38.

Parvilucina approximata, Hendrickx \& Toledano, 1994: 12; Coan et al., 2000: 265 , pl. 50

Liralucina approximata, Coan\& Scott, 2012:346.

Concha pequeña redondeada subequilateral, picos pronunciados y lúnula lanceolada; escultura es cancelada, presenta costillas radiales con interespacios estrechos, y costillas concéntricas más separadas; en las áreas anterior y posterior desaparecen las costillas radiales y las concéntricas se levantan formando lamelas bajas; no se aprecian restos de periostraco; la charnela es típica del género, con dos dientes cardinales y dos laterales en cada valva. El color externo e interno es blanco y los márgenes internos de las valvas carinados. Longitud $6,3 \mathrm{~mm}$; altura $5,8 \mathrm{~mm}$.

Distribución: Santa Barbara, California, a través del Golfo de California (Coan et al. 2000), hacia el sur hasta Panamá (Keen 1971; y la Isla de la Plata, Ecuador (Shasky 1984).

Localidades: Piura (Bahía de Sechura).

Habitat: Fondo arenoso, desde el intermareal hasta los $86 \mathrm{~m}$.

Material examinado: 1 lote, 1 especimen (LaBSIM).

Observaciones: Nuevo registro para el mar peruano.

Superfamilia Carditoidea J. Fleming, 1828

Familia Carditidae J. Fleming, 1828

Subfamilia Carditamerinae Chavan, 1969

Genero Carditamera Conrad, 1838

Carditamera radiata (G.B. Sowerby I, 1833)

Figura 5

Cardita radiata G.B. Sowerby I, 1833: 195.

Carditamera radiata, Dall 1909: 261; Skoglund, 2001: 43.

Cardita (carditamera) radiata, Hertlein and Strong 1946: 108; Keen, 1971: 107, fig. 238 


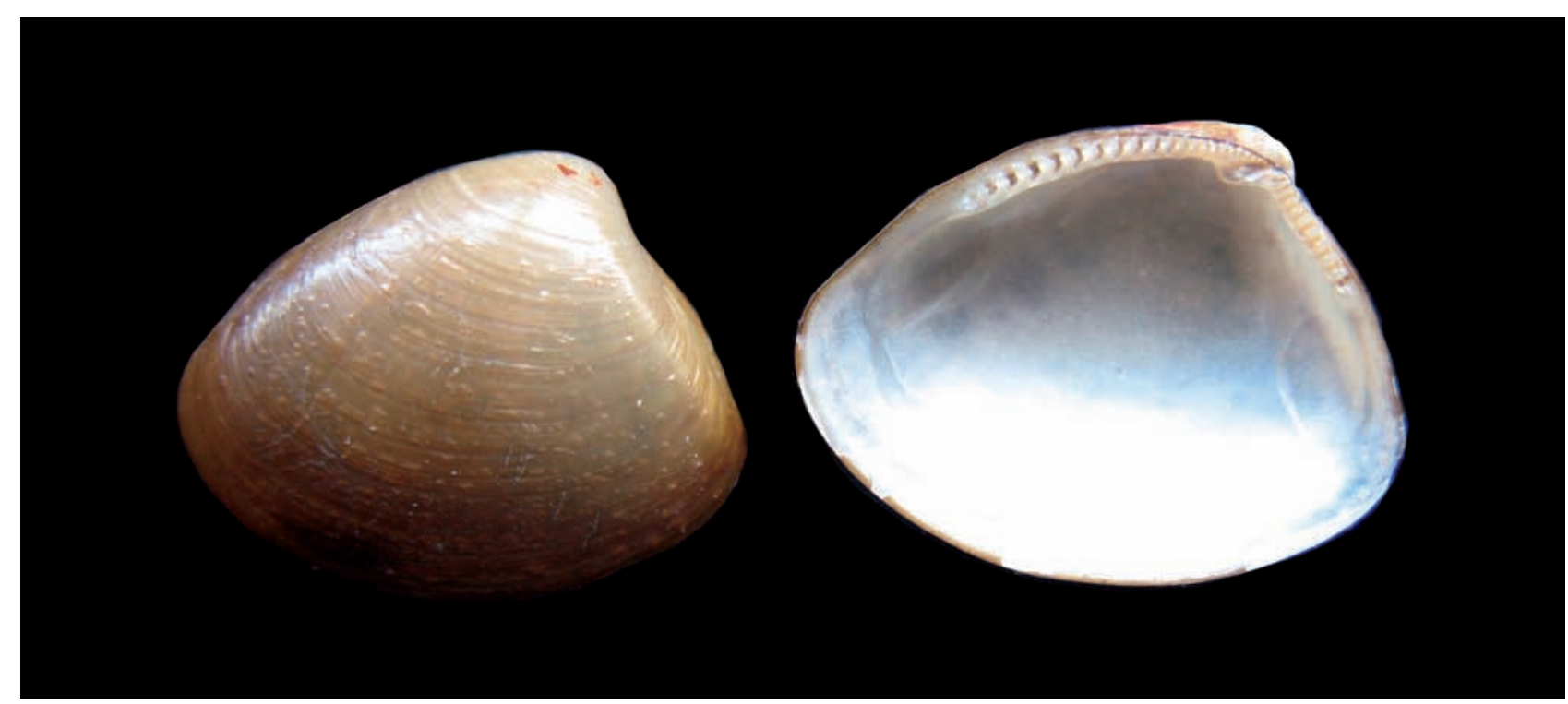

Figura 1. Ennucula panamina (Dall, 1908), Longitud; $22,0 \mathrm{~mm}$; altura $16,0 \mathrm{~mm}$.

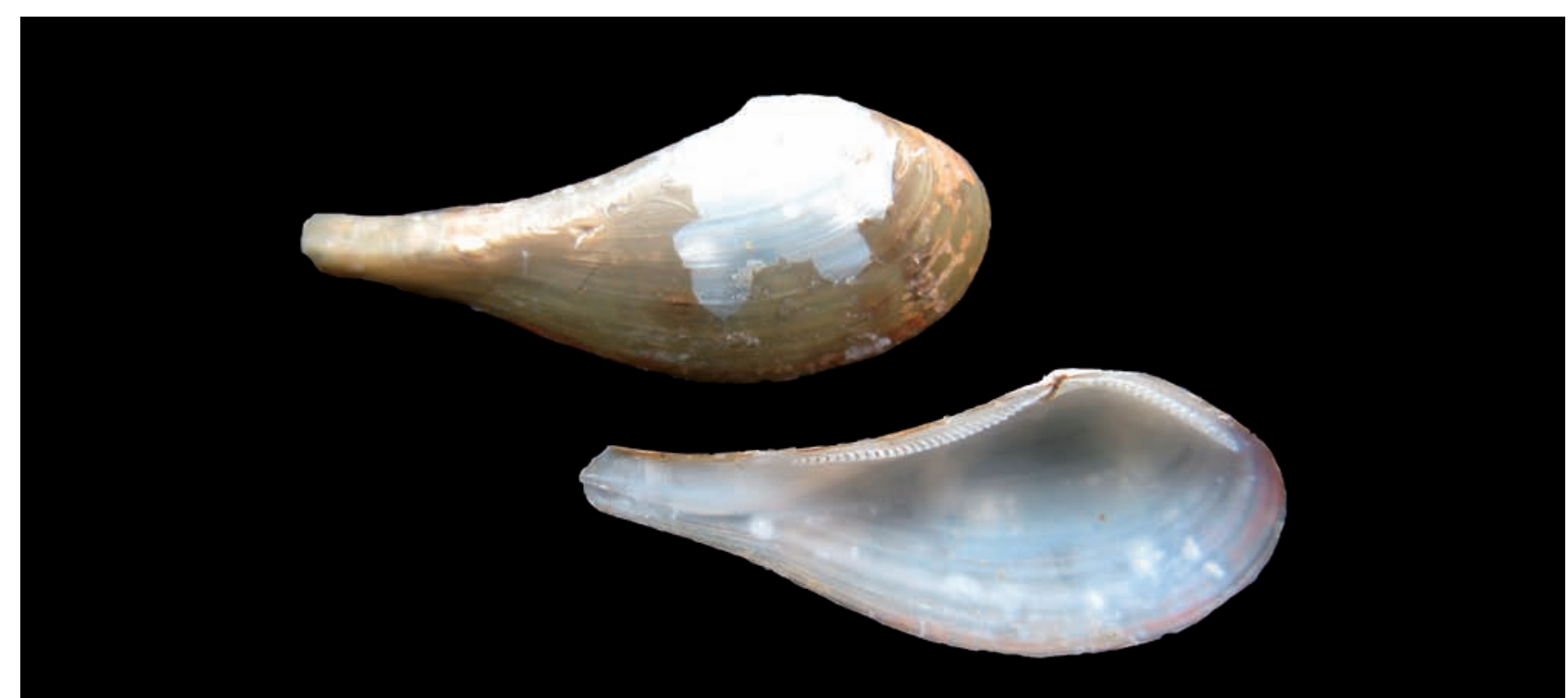

Figura 2. Nuculana (Nuculana) loshka, (Dall,1908); Longitud, 17,0 mm; altura, 7,0 mm.

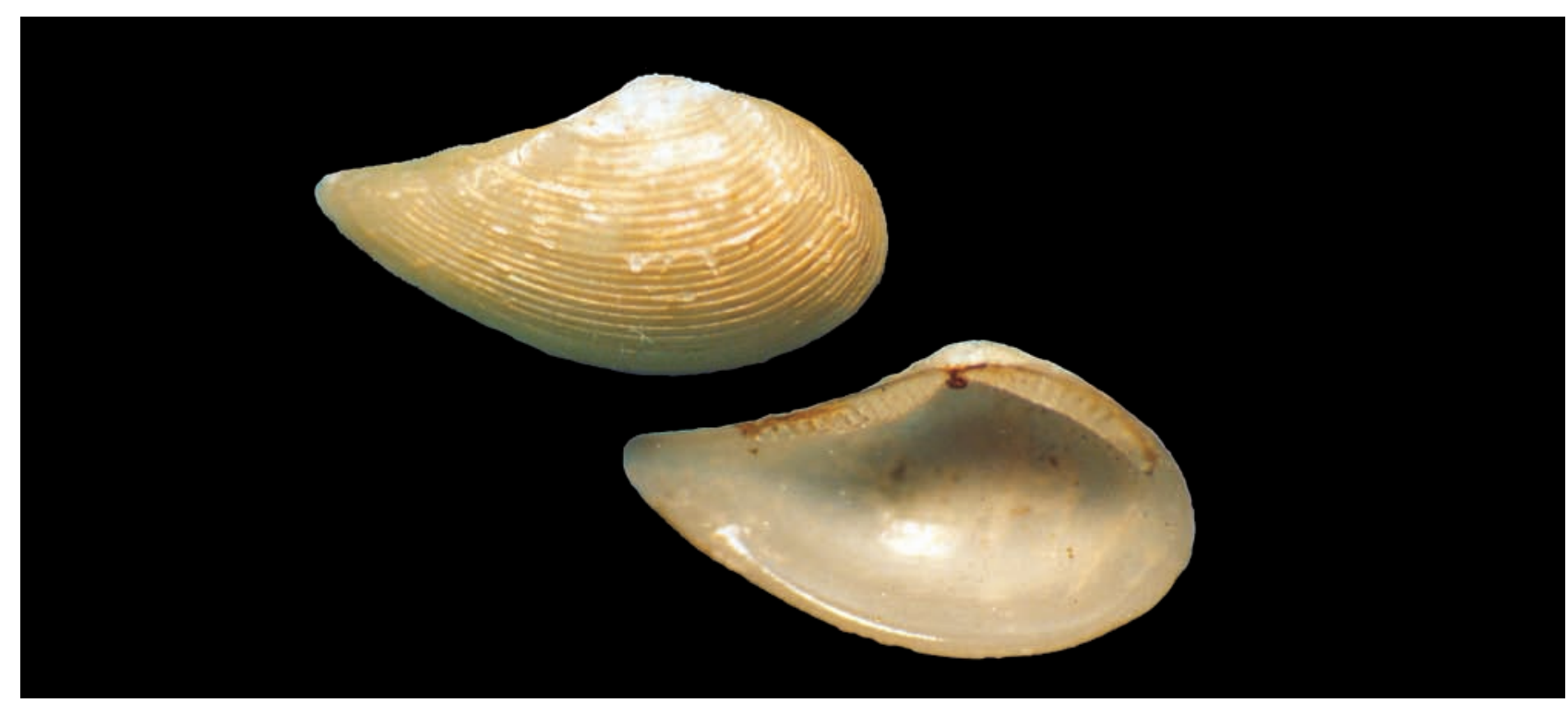

Figura 3. Saccella laeviradius (Pilsbry \& Lowe, 1932); Longitud, 6,2 mm; altura, 3,3 mm. 
Carditamera (Carditamera) radiata, Olsson 1961: 189, pl. 26, figs. 1- 1c.

Carditamera radiata, Bernard, 1983: 34; Cruz \& Jiménez, 1994: 38, fig. 19.

Concha alargada, rectangular, inequilateral con los umbos cerca del extremo anterior cuyo borde es redondeado, parte posterior con bordes dorsal y ventral casi paralelos, el borde posterior inclinado dorsalmente es irregular por las proyecciones de las costillas; con 17 costillas radiales con interespacios amplios, las anteriores son finamente noduladas, las del disco oblicuamente aplanadas, y las posteriores mas gruesas, redondeadas y escamosas; charnela con dos dientes cardinales y dos laterales en cada valva. El color externo es blanco amarillento con manchas oscuras sobre las costillas; interior blanco con manchas pardo claro, el ejemplar juvenil presenta intensas manchas marrón oscuras en la parte inferior del disco y en el área posterior, los bordes internos son crenulados. Longitud $14,0 \mathrm{~mm}$; altura $6,5 \mathrm{~mm}$.

Distribución: Baja California, México hasta Guayaquil, Ecuador (Keen 1971).

Localidades: Tumbes (Zarumilla, El Rubio, Máncora).

Habitat: Fondo fangoso.

Material examinado: 3 lots, 7 especimens (LaBSIM).

Observaciones: Nuevo registro para el mar peruano.

Genero Carditella Smith, 1881

Subgenero Carditella, s.S.

Carditella naviformis (Reeve, 1843)

Figura 6

Cardita naviformis Reeve, 1843:194; Cardita pl. 9, fig. 45

Cardita (Glans) naviformis, Dall, 1909: 261.

Carditella naviformis, Ramorino, 1968: 203-204, pl. 2, figs. 3, 4; pl. 6 , figs. 1,4 .

Carditella (Carditella) naviformis, Bernard, 1983: 34.

Carditella tegulata, Dall,: 1909: 262; Soot-Ryen, 1959: 40, lám. 1, fig.11 (no de Reeve); Marincovich, 1973: 10, fig. 9; Paredes, 1974: lám. 14, fig. 1; Bernard, 1983: 34; Alamo \& Valdivieso, 1987: 126, fig. 269; Guzmán et al., 1998:68, fig.85; Paredes et al. 1999:38.

Concha pequeña, sólida, trapezoidal, inequilateral con los umbos anteriores, los bordes dorsal posterior y ventral son casi paralelos, el borde anterior algo cóncavo en la región umbonal y redondeado en el resto, el borde posterior ampliamente redondeado; charnela con ligamento externo y un pequeño resilio, valva derecha con tres dientes cardinales, un lateral anterior y otro posterior, la valva izquierda con dos cardinales y dos laterales; escultura con 11 a 13 costillas radiales prominentes de aspecto nodular escamoso, los interespacios son anchos y algo profundos; periostraco pardo claro y color externo de la concha blanco amarillento, interior blanco a veces con manchas café y con los surcos producidos por la escultura externa, los bordes internos son crenulados. Longitud 5,0 mm; altura 2,8 $\mathrm{mm}$.

Distribución: Iquique (Marincovich 1973) al Estrecho de Magallanes (Soot-Ryen 1959); Arica, a Magallanes e Islas Malvinas (Ramorino 1968).

Localidades: Ancash (Playa Tortugas), Lima (Ancón).

Habitat: En grietas de las rocas,intermareal, y submareal. Hasta 260 m de profundidad (Bernard 1983).
Material examinado: 2 lotes, 38 especímenes.

Observaciones: Nuevo registro para el mar peruano.

Superfamilia TELLINOIDEA BlainVILLE, 1814

FAMILIA TELLINIDAE BLAINVILLE, 1814

Subfamilia Macominae Olsson, 1961

Genero Macoma Leach, 1819

Subgenero Psammacoma Dall, 1900

Macoma (Psammacoma) elytrum Keen, 1958

Figura 7

Macoma (Psammacoma) elytrum Keen 1958: 244, pl. 30, fig. 14; 1971: 227, fig. 562; Abbott, 1974: 507; Bernard, 1983: 45; Hendrickx \& Toledano, 1994: 20; Skoglund, 2001: 71.

Macoma (Psammacoma) elongata, Hertlein \& Strong, 1949: 89.

Psammacoma (Psammacoma) elytrum, Olsson, 1961: 415, pl. 74, fig. 7.

Macoma elytrum, Gemmell et al., 1987: 51.

Concha delgada y alargada, subrectangular; el lado anterior más largo alcanza casi los dos tercios de la longitud total y tiene el borde redondeado, el borde dorsal anterior es recto y el posterior oblicuo forma un ángulo con el borde posterior cuadrado y reflejado hacia la derecha,el cual queda abierto; la región umbonal es algo inflada y los picos pequeńos son opistogirados, una suave área radial deprimida baja desde la región umbonal hasta el borde inferior de cada valva; solamente se observan finas líneas de crecimiento, mas notorias cerca de los bordes inferiores de las valvas; la charnela presenta dos dientes cardinales en cada valva; el periostraco es pardo claro, liso y brillante; el seno paleal es amplio, profundo y elevado dorsalmente y los bordes de las valvas lisos. Color externo blanco amarillento y el interior blanco translúcido. Longitud 27,8 $\mathrm{mm}$; altura $14,0 \mathrm{~mm}$.

Distribución: Baja California a través del Golfo de California México, hasta Ecuador (Keen 1971).

Localidades: Tumbes (Punta La Cruz, Caleta Cruz).

Habitat: Fondo arenoso, 25 hasta $36 \mathrm{~m}$.

Material examinado: 2 lotes, 2 especímenes (LaBSIM).

Observaciones: Esta especie fue determinada por Dr. Eugene Coan. Nuevo registro para el mar peruano.

Genero Psammotreta Dall, 1900

Subgenero PSAMMOTRETA, s.s.

Psammotreta (Psammotreta) pura (Gould, 1853)

Figura 8

Tellina pura Gould, 1853: 398, pl. 16, fig. 3.

Macoma (Psammotreta) pacis, Hertlein \& Strong, 1949: 92-93.

Psammotreta gubernacula, Olsson, 1961: 413, pl. 74, fig. 4, 4

Psammotreta (Psammotreta) viridotincta, Keen, 1971: 231, fig. 572; Gemmell et al., 1987: 51; Skoglund, 2001: 72.

Psammotreta (Psammotreta) pura, Bernard, 1983: 45.

Concha aovada, delgada y translúcida, algo comprimida, subequilateral, lado anterior más alargado con borde ampliamente redondeado; lado posterior corto, deprimido, con borde redondeado, y las valvas flexionadas hacia la derecha; charnela con dos dientes cardinales en cada valva y el ligamento hundido casi alcanza el borde de la lámina articular bajo y detrás de los picos; escultura consiste en estrías conmarginales muy finas, que están 

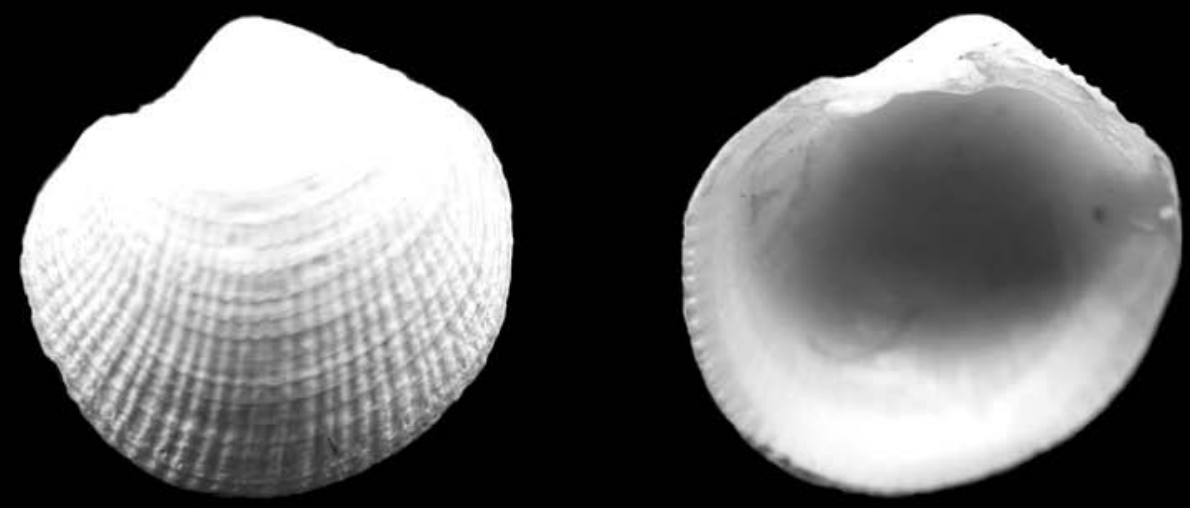

Figura 4. Liralucina approximata (Dall, 1901); Longitud, 6,3 mm; altura, 5,8 mm.

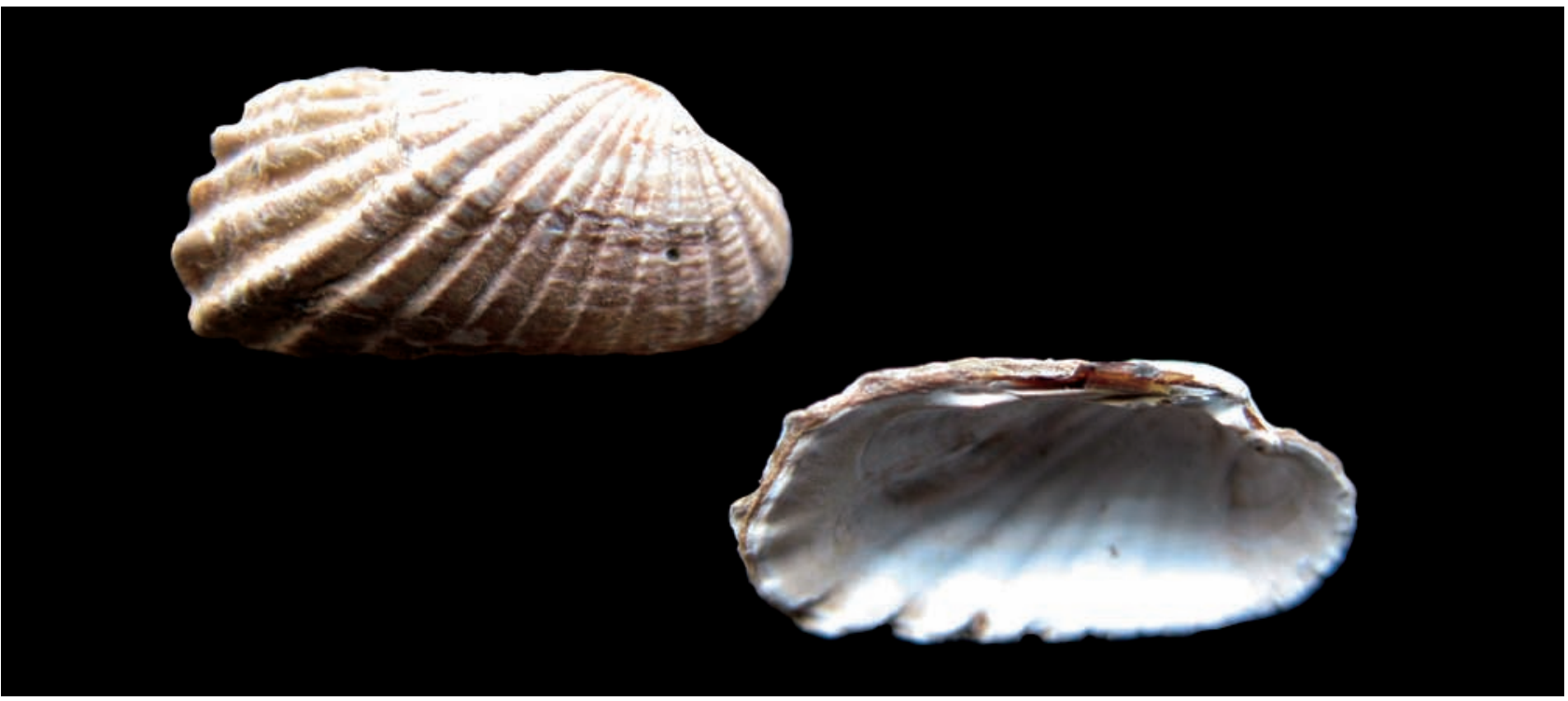

Figura 5. Carditamera radiata (G. B. Sowerby I, 1833); Longitud, 14,0 mm; altura, 6,5 mm.

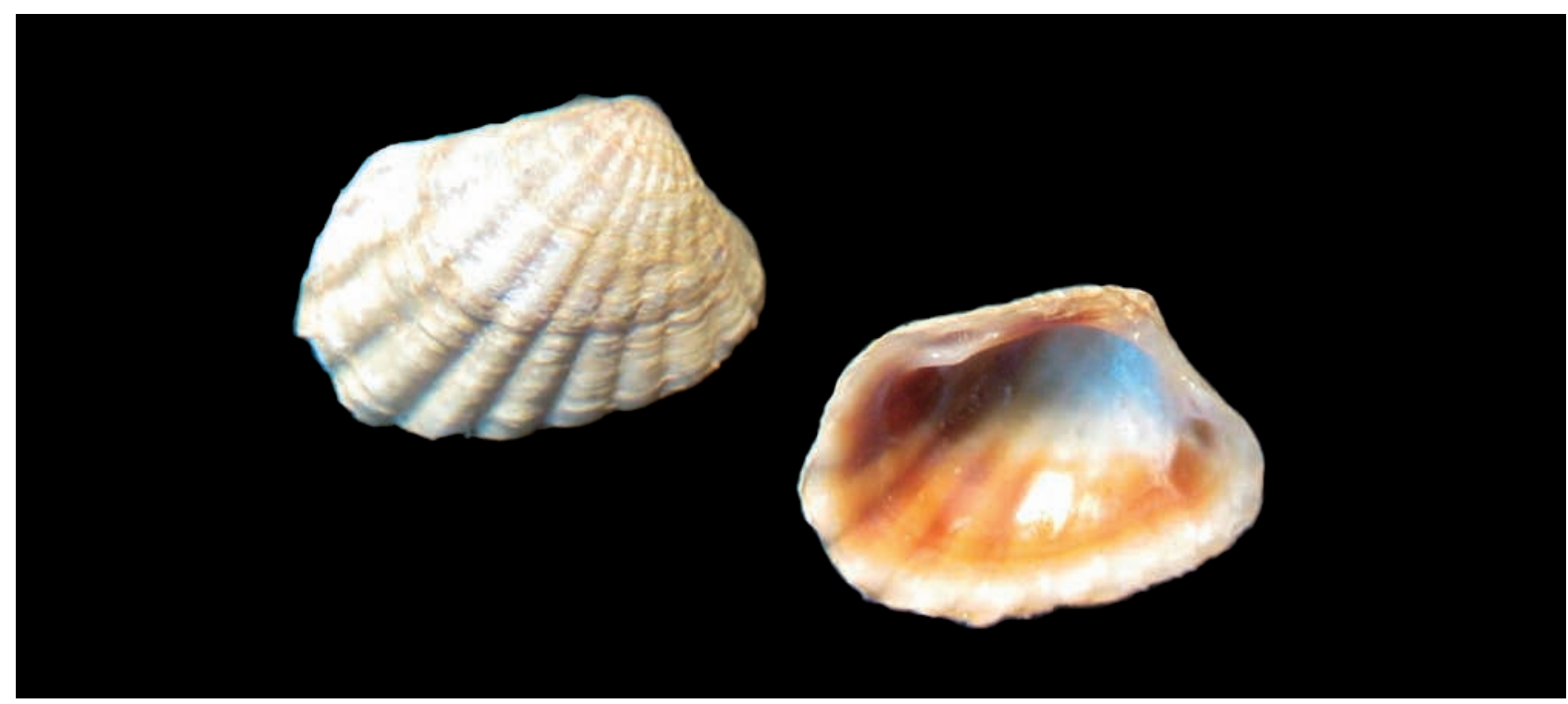

Figura 6. Carditella (Carditella) naviformis (Reeve, 1843); Longitud, 5,0 mm; altura, 2,8 mm. 
interrumpidas por algunas líneas de crecimiento ampliamente separadas; color externo blanco mate con manchas amarillentas, interior blanco brillante con tonalidad amarillenta en la cavidad umbonal; seno paleal amplio, anguloso dorsalmente y con extremo redondeado, bordes de las valvas lisos. Longitud $15,3 \mathrm{~mm}$; altura 10,5 mm (ejemplares juveniles, procedentes de Sechura).

Distribución: San Felipe, Baja California, México (Gemmell et al. 1987) hasta Puerto Limones, Ecuador (Olsson 1961).

Localidades: Piura (Sechura), Arequipa (Matarani).

Habitat: Fondo de arena gruesa y grava, $50 \mathrm{~m}$ de profundidad.

\section{Material examinado: 2 lotes, 5 especimenes (LaBSIM).}

Observaciones: Según Olsson (1961), la especie presenta mucha variación en su forma de manera que algunos ejemplares son más cuadrados u oblongos. La determinación taxonómica la realizó el Dr. Eugene Coan. Nuevo registro para el mar peruano.

ORdEN MYOIDA

SUBORDEN MYINA

Superfamilia Myoidea Lamarck, 1809

FAMILIA MYIDAE LAMARCK, 1809

Subfamilia Cryptomyinae Habe, 1977

Genero Sphenia Turton, 1822

Sphenia fragilis ( $\mathrm{H}$. Adams and A. Adams, 1854)

Figura 9

Tyleria fragilis H. Adams and A. Adams, 1854: 418; 1856: 368, pl. 97, figs. 3, 3a.

Sphenia fragilis, Olsson, 1961: 424-425, pl. 77, figs. 9-9b; Keen, 1971: 273-274, fig. 673; Abbott, 1974: 537, fig. 5990; Hendrickx \& Toledano, 1994: 25; Coan, 1999: 107, 109, 110, figs. 2-6, 18 , 24; Coan et al., 2000: 474-476, pl. 101; Skoglund, 2001: 82.

Sphenia luticola, Bernard, 1983: 58; Gemmel et al., 1987: 58, fig. 71; Cruz y Jiménez, 1994: 92, fig. 072.

Concha pequeña delgada y alargada, umbo más cercano al borde anterior redondeado, parte anterior ligeramente más inflada y la posterior algo estrecha termina truncada; charnela presenta en la valva izquierda un gran condróforo proyectado, y el resilio se inserta en el interior del pico de la valva derecha; escultura consiste en finas costillas conmarginales mas desarrolladas en la vertiente posterior, y el crecimiento irregular ha deformado la superficie de la conchilla; el color es blanco translúcido y el periostraco parduzco se proyecta más allá del borde de la conchilla en el extremo posterior. Interior blanquecino brillante y bordes de las valvas lisos. Longitud 5,0 $\mathrm{mm}$; altura 2,8 $\mathrm{mm}$.

Distribución: Carpinteria, Santa Barbara County, California, a través del Golfo de California, hacia el sur hasta Salinas, Provincia de Guayas, Ecuador. Oeste del Atlántico, desde la Isla Hunting State Park, South Carolina, Saint Lucie e Isla Sanibel, Florida; y al sur Isla Padre, Texas, hasta Bahia Maldonado, Uruguay (Coan et al. 2000).

Localidades: Tumbes (Puerto Pizarro).

Habitat: Cavidades de las raíces de los mangles.

Material examinado: 1 lote, 1 especimen (LaBSIM).

Observaciones: Si los reportes de Olsson (1961) y de Keen (1971) fueron fueron puestos en duda (Coan 1999), el hallazgo de este especimen vivo confirma la presencia de la especie en el mar peruano. Nuevo registro para el mar peruano.

\section{Familia Corbulidae Lamarck, 1818 \\ SubFAmILIA CoRBULINAE LAMARCK, 1818 \\ Genero Corbula Bruguière, 1797 \\ Subgenero Caryocorbula Gardner, 1926}

\section{Caryocorbula amethystina Olsson, 1961}

\section{Figura 10}

Caryocorbula (Caryocorbula) amethystina Olsson, 1961: 429, pl. 75, figs. 1-1c.

Corbula (Caryocorbula) amethystina, Keen, 1971: 264, fig. 674; Cruz 1983: 754, pl. 24, figs. 67, 67a; Bernard, 1983: 58; Skoglund, 2001: 82; Coan, 2002a: 51-52, figs. 1, 39.

Nuestros ejemplares son juveniles, pues esta especie puede exceder los 27 mm de longitud (Olsson 1961, Keen 1971). La concha es sólida algo alargada y de forma oblicua, los picos son subcentrales y los umbos bajos, el extremo anterior es más corto

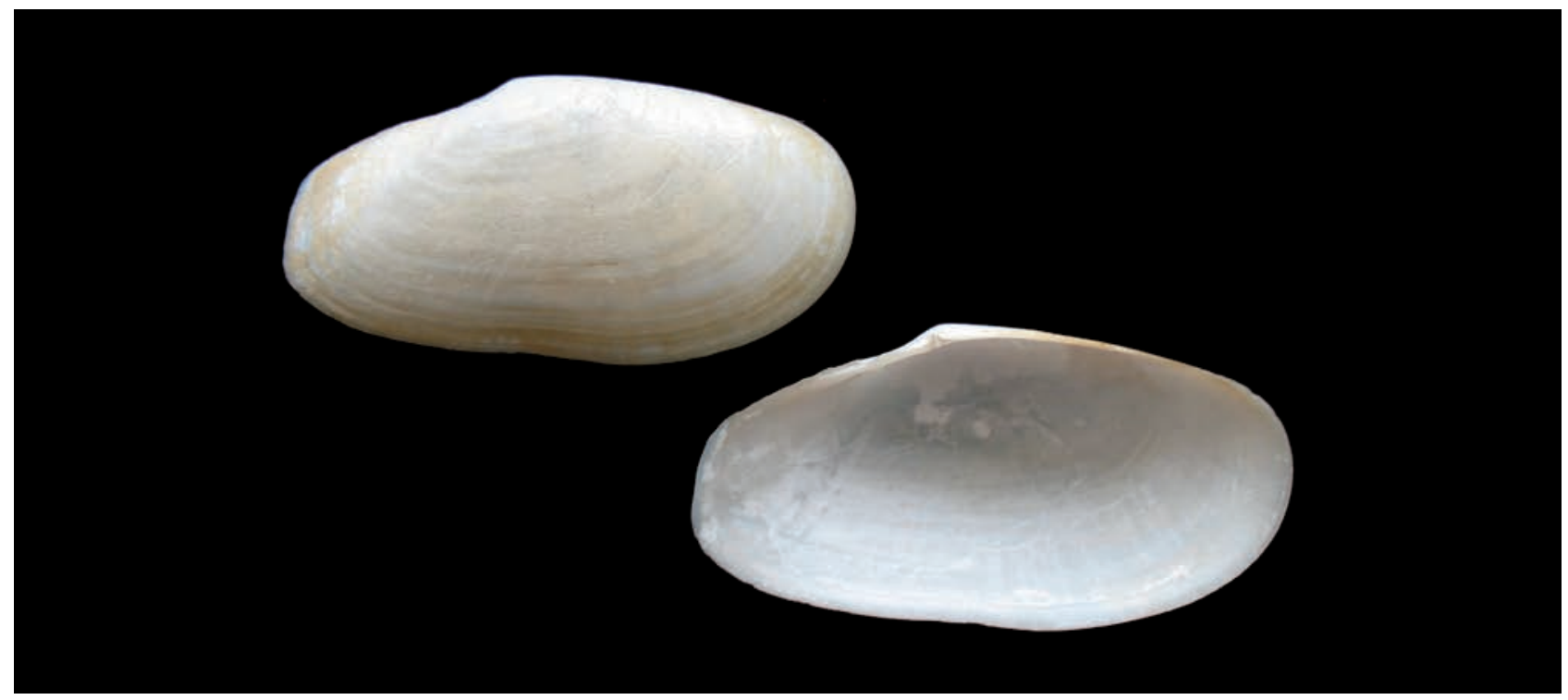

Figura 7. Macoma (Psammacoma) elytrum Keen, 1958; Longitud, 27,8 mm; altura, 14,0 mm. 


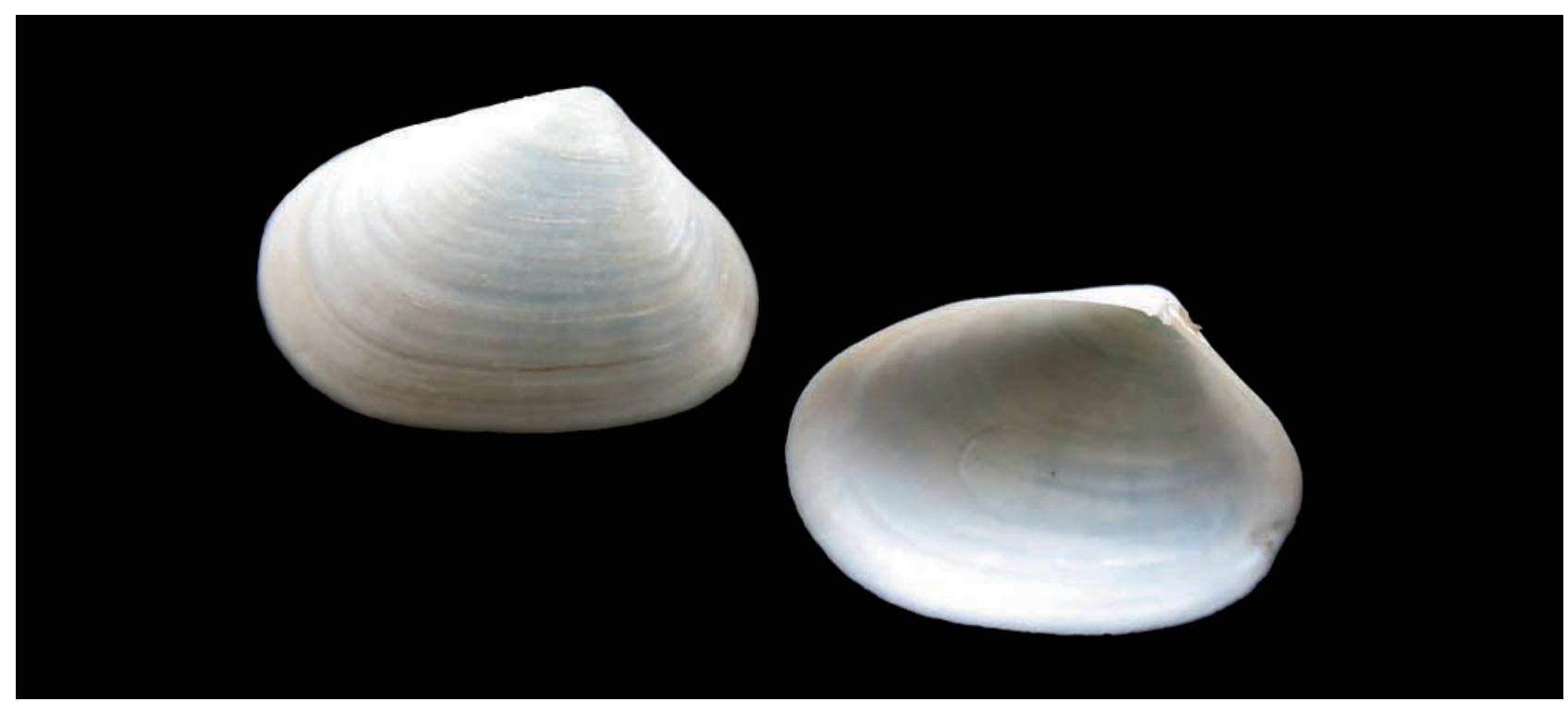

Figura 8. Psammotreta (Psammotreta) pura (Gould, 1853); Longitud, 15,3 mm; altura, 10,5 mm (ejemplares juveniles, procedentes de Sechura).

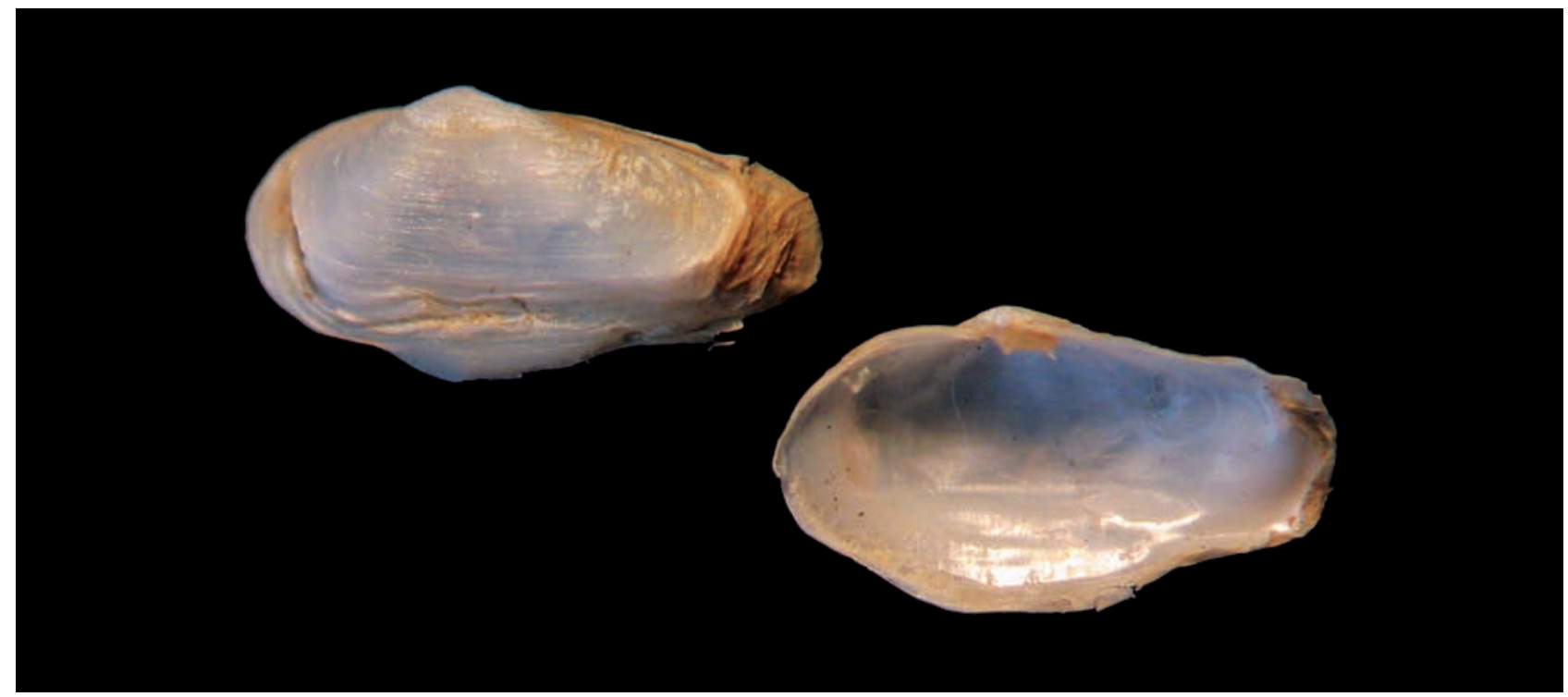

Figura 9. Sphenia fragilis (H. Adams \& A. Adams, 1854); Longitud, 5,0 mm; altura, 2,8 mm.

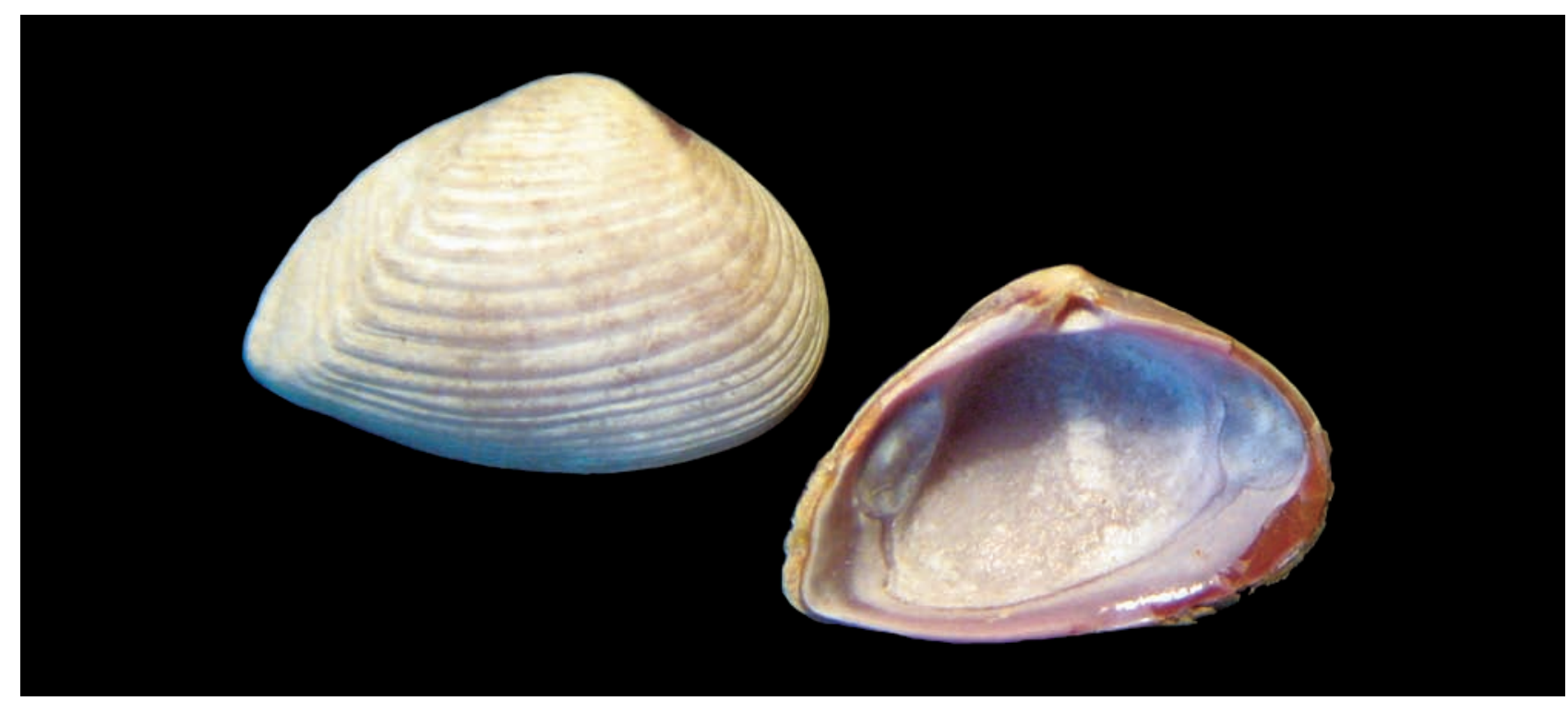

Figura 10. Caryocorbula amethystina Olsson, 1961; Longitud, 7,0 mm; altura, 4,7 mm. 
y su borde es redondeado; el lado posterior se estrecha hacia el borde ventral terminando en un extremo punteado romo, un lomo anguloso delimita la vertiente posterior; escultura con anchas y bajas costillas redondeadas muy juntas y algo oblicuas en el umbo y en el disco, se suavizan y se convierten en líneas en las vertientes anterior y posterior así como cerca al borde ventral de la valva; charnela tiene un ligamento interno delgado y en la valva derecha un gran diente cardinal que encaja en el condróforo de la valva izquierda; las inserciones de los músculos aductores y la línea paleal entera nítidamente marcadas, los bordes de las valvas lisos. Longitud 7,0 $\mathrm{mm}$; altura 4,7 $\mathrm{mm}$.

Distribución: Mazatlán, Sinaloa, México, hasta Playas [de Villamil], Guayas, Ecuador (Coan 2002a).

Localidades: Piura (Bahía de Sechura).

Habitat: Fondo arenoso.

Material examinado: 1 lote, 2 especímenes (LaBSIM).

Observaciones: Nuevo registro para el mar peruano.

\section{Agradecimientos}

Expresamos nuestro especial agradecimiento al Doctor Eugene V. Coan y a P. Scott, investigadores del Museo de Santa Bárbara, California, por su ayuda en la determinación taxonómica. Asimismo, agradecemos al Consejo Superior de Investigaciones por su valioso apoyo a los estudios de nuestra biodiversidad malacológica marina.

\section{Literatura citada}

Abbott R.T. 1974. American Seashells. The Marine Mollusca of the Atlantic and Pacific coasts of North America. 2 ed. Van Nostrand Reinhold Company. New York. 663 pp.

Bernard F.R., 1983. Catalogue of the living bivalvia of the Eastern Pacific Ocean: Bering Strait to Cape Horn. Canadian Special Publication of Fisheries and Aquatic Sciences 61: $102 \mathrm{pp}$.

Coan E.V. 1999. The eastern Pacific species of Sphenia (Bivalvia: Myidae). The Nautilus 113(4): 103-120.

Coan E.V. 2002. The Eastern Pacific recent species of the Corbulidae (Bivalvia). Malacologia 44(1): 47-105.

Coan E.V., P. Scott \& F. R. Bernard. 2000. Bivalve Seashells of Westewrn North America: Marine Bivalve Mollusks from Artic Alaska to Baja California. Santa Barbara Museum of Natural History Monographs Number 2: 764 p.

Coan E.V. \& P. Scott. 2012. Bivalve seashells of tropical West America: marine bivalve mollusks from Baja California to Northern Peru. Santa Barbara Museum of Natural History Monographs Number 6 and Studies in Biodiversity Number 4. 1258pp.

Cruz M. 1983. Bivalvos del Golfo de Guayaquil. Pub. INOCAR, Ecuador 2(2): 735-819, 38 láms.

Cruz R.A. \& J.A. Jimenez. 1994. Moluscos asociados a las áreas de manglar de la Costa Pacífica de América Central: Guia. Heredia, C.R.: EFUNA. 182 pp.

Dall W.E. 1908. Reports on the dredging operations off the west coast of Central America to the Galapagos, to the west coast of Mexico, and in the Gulf of California.... XIV. The Mollusca and Brachiopoda. Bull. Mus. Comp. Zool. 43: $205-487$

Dall W.H. 1909. Report on a collection of shells from Perú, whith a summary of the littoral marine Mollusca of the Peruvian Zoological Province. Proc. U.S.N. Mus. 37(1704): 147-294.
Gemmell J., B.W. Myers \& C.M. Hertz. 1987. A faunal study of the bivalves of San Felipe and environs, Gulf of California, from the Gemmell Collection (1965 to 1976). Festivus 18(Supplement): 1-72.

Guzmán N., S. Saá \& L. Ortlieb. 1998. Catálogo descriptivo de los moluscos litorales (Gastropoda y Pelecypoda) de la zona de Antofagasta, $23^{\circ} \mathrm{S}$ (Chile).

Hendrickx M.E. \& A. Toledano. 1994. Catálogo de moluscos pelecypodos, gasteropodos y poliplacoforos. Colección de Referencia. Estación Mazatlán, ICML, UNAM. Comisión Nacional para el Conocimiento y Uso de la Biodiversidad e Instituto de Ciencias del Mar y Limnología. UNAM, México. $71 \mathrm{pp}$.

Hertlein L.G. \& A.M. Strong. 1940-1950. Eastern Pacific Expeditions of the New York Zoological Society. Mollusks from the West Coast of Mexico and America Central. Parts I-IX. Zoologica, New York Zoological Society, vols. 25-35.

Keen A.M. 1971. Sea shells of tropical West America. 2a. ed. Stanford Univ. Press, California, 1064 pp., 3325 figs. 22 pls.

Marincovich L. Jr. 1973. Intertidal Mollusks of Iquique, Chile. Natural History Museum of Los Angeles County Science Bulletin 16:1-49, figs. 1-102.

Olsson A.A. 1961. Mollusks of the tropical eastern Pacific. Paleonto. Res. Ins. Ithaca, New york. 574 pp., pls 1-86.

Paredes C. \& F. Cardoso. 1998. Nuevos registros de gasterópodos para el litoral peruano. Rev. peru. biol.. 5 (2): 118-122

Paredes C., P. Huaman, F. Cardoso, R. Vivar \& V. Vera. 1999. Estado actual del conocimiento de los moluscos acuáticos en el Perú. Rev. peru. biol. 6(1): 5-47

Paredes, C., F. Cardoso, K. Altamirano, P. Baltazar \& L. Romero. 2010. La familia Conidae en el mar peruano. Rev. peru. biol. 17(1): 065-073.

Paredes C. \& F. Cardoso. 2001a. Nuevos registros de Protobranchia (Mollusca: Bivalvia) para el mar peruano. Rev. per. Biol. 8(1): 5-10

Paredes C. \& F. Cardoso. 2001b. El género Donax en la Costa Peruana (Bivalvia: Tellinoidea). Rev. per. Biol. 8(2): 83-93

Paredes C. \& F. Cardoso. 2003. Adiciones a los moluscos bivalvos marinos del Perú. Rev. per. biol. 10(1): 53-58

Paredes C. \& F. Cardoso. 2007. La Familia Calyptraeidae en el Perú. Rev. perú. biol. 13 (3): 177-184

Paredes C. \& F. Cardoso. 2008. Nuevos registros de bivalvos marinos para el Perú. Rev. per. biol. 15(1): 11-14

Paredes C. \& F. Cardoso. 2009. Nuevos registros de gasterópodos para el mar tropical del Perú. Rev. peru. biol. 15(2): 073078.

Paredes C., A. Indacochea, F. Cardoso \& K. Ortega. 2005. Familia Ellobiidae (Gastropoda: Archaeopulmonata) en el litoral peruano. Rev. peru. biol. 12(1): 69-76.

Ramírez R., C. Paredes y J. Arenas. 2003. Moluscos del Perú. Rev. Biol. Trop. (Suppl. 3): 225-284.

Ramorino L. 1968. Pelecypoda del fondo de la Bahía de Valparaiso. Rev. Biol Mar. 13(3): 175-285, pls. 1-10.

Shasky D.R. 1984. A preliminary checklist of marine mollusks from Manabí Province, Ecuador. The Western Society of Malacologists Annual Report [for 1983] 16: 25-32.

Skoglund C. 2001. Panamic Province Molluscan literature. Additions and changes from 1971 through 2000. I. Bivalvia. The Festivus 32 (Suppl.): 1-119.

Soot-Ryen T. 1959. Pelecypoda. Rep. Lund. Univ. Chile. Exped. 1948-1949, 35: 1-86. 